\title{
Density and isospin-asymmetry dependence of high-momentum components
}

\author{
A. Rios,,${ }^{1, *}$ A. Polls, ${ }^{2}$ and W. H. Dickhoff ${ }^{3}$ \\ ${ }^{1}$ Department of Physics, Faculty of Engineering and Physical Sciences, University of Surrey, Guildford, Surrey GU2 7XH, United Kingdom \\ ${ }^{2}$ Departament d' Estructura i Constituents de la Matèria, Universitat de Barcelona, E-08028 Barcelona, Spain \\ ${ }^{3}$ Department of Physics, Washington University, St. Louis, Missouri 63130, USA
}

(Received 2 January 2014; revised manuscript received 7 March 2014; published 4 April 2014)

\begin{abstract}
We study the one-body momentum distribution at different densities in nuclear matter, with special emphasis on its components at high momentum. Explicit calculations for finite neutron-proton asymmetry, based on the ladder self-consistent Green's function approach, allow us to access the isospin dependence of momentum distributions and elucidate their role in neutron-rich systems. Comparisons with the deuteron momentum distribution indicate that a substantial proportion of high-momentum components are dominated by tensor correlations. We identify the density dependence of these tensor correlations in the momentum distributions. Further, we find that highmomentum components are determined by the density of each subspecies and we provide a new isospinasymmetry scaling of these components. We use different realistic nucleon-nucleon interactions to quantify the model dependence of our results.
\end{abstract}

DOI: 10.1103/PhysRevC.89.044303

PACS number(s): 13.75.Cs, 24.10.Cn, 21.65.Mn, 21.30.-x

\section{INTRODUCTION}

Short-range correlations (SRCs) have been unambiguously identified in a variety of nuclear and hadronic physics experiments [1-8]. Their presence is subtle at the one-body level because they occur at large missing energy and not near the Fermi energy [3]. At the two-body level, these correlations must occur at all energy scales on account of the expected reduction of the amplitude for nucleons to be found at small relative distances. Ultimately, these two-body correlations are also responsible for the high-momentum components of the single-particle distribution. The quantitative structure of the neutron and proton high-momentum components can now be accessed experimentally [4,6,7]. A clear manifestation of these correlations is the population of high-momentum components of the single-particle momentum distribution $[9,10]$. The existence of such components, in turn, involves a necessary depletion of the low-momentum single-particle strength to conserve the total density [11]. Green's function techniques are ideally suited to study such effects, as they can incorporate SRCs via ladder summation techniques in a self-consistent Green's function (SCGF) description [12], including a complete treatment of off-shell effects. Moreover, many-body approximations can be formulated to preserve basic conservation laws, including the density normalization [13].

Experiments indicate that two-body correlations are dominated by neutron-proton components [6]. This suggests that tensor correlations, in addition to SRCs, can play a role in the high-momentum structure of the nuclear wave function

\footnotetext{
*a.rios@surrey.ac.uk
}

Published by the American Physical Society under the terms of the Creative Commons Attribution 3.0 License. Further distribution of this work must maintain attribution to the author(s) and the published article's title, journal citation, and DOI.
[14]. Tensor correlations and SRCs act in somewhat different momentum regions, however. A distinction between the two is presumably possible if suitable momentum cuts are applied. One expects that a sizable population in the neutron-proton (np) two-body density matrix translates into a relatively large population in the one-body momentum distribution. Theoretical calculations of the latter are challenging, as one needs to treat and model nuclear short-range components within a many-body environment.

Revisiting theoretical descriptions of high-momentum components is particularly timely, in view of the recently found correlation between the EMC effect and SRCs [15-17]. Data are now available for a relatively large selection of nuclei, which has allowed for the empirical determination of the density dependence of parameters that quantify SRCs. Whereas there is still a discussion regarding two-body centerof-mass (and other) corrections in establishing the connection between theory and experiment $[16,18]$, the qualitative guidance generated by such experiments is very valuable. We discuss the density dependence of such correlations using numerical data for both isospin symmetric and asymmetric nuclear matter. In particular, we discuss the impact of a finite isospin polarization on SRCs. We also highlight the importance of tensor components in a relatively high-momentum region.

SRCs in nuclear matter have often been studied in connection to the correlated nucleon momentum distribution. High-momentum components in the medium are indeed a consequence of SRCs. A variety of methods have been employed to study these distributions, ranging from correlated basis functions [19] to quantum Monte Carlo studies [20]. In particular, extensive studies have been carried out using Brueckner-Hartree-Fock $G$ matrices as starting points [21], including off-shell corrections. Recent contributions within this extended Brueckner-Hartree-Fock approach have tackled symmetric [22] and asymmetric nuclear matter [23].

In addition, several groups have recently tried to provide scaling arguments for the effect of SRCs in nuclei [18,24-27]. 
In practice, one aims at identifying how these effects change with particle number and if they are dominated by the number of particles or by the number of pairs. From an infinite-matter perspective, if one stays at the one-body level, total and partial nucleon densities are the more obvious available quantities for such a scaling. In the following, we illustrate how the two are connected in the SCGF approach. We base our analysis on the isospin-asymmetry dependence of the dilute Fermi gas (DFG) in the high-momentum limit (see the Appendix for details) $[28,29]$. We limit our discussion to the one-body momentum distribution, but note that the two-body distribution can be accessed in our formalism [30].

The variation of the results with the underlying nucleonnucleon $(N N)$ interaction provides an indication of the robustness of the physical effects at play. We choose four $N N$ forces, with very different short-range and tensor components, to explore the widest possible range. We thereby aim to reduce model dependence and to identify trends within our many-body summation technique. The Argonne v18 (Av18) represents a phenomenological parametrization in real space, including 18 spin-isospin operators [31]. It is a typical example of a particularly strong, but finite, short-range core. The charge-dependent Bonn (CD-Bonn) interaction is based on a meson-exchange picture and provides a very accurate fit to the two-body sector [32]. Its short-range core is softer than Av18, which yields less high-momentum components. We also use the Idaho next-to-next-to-next-to-leading-order (N3LO) chiral-perturbation-theory potential of Ref. [33]. Its low-energy constants are fitted to reproduce the two-body sector with a large accuracy in a $\chi^{2}$ procedure (see Ref. [34] for an alternative fitting strategy). Owing to its very nature, as an effective-field-theory potential, the interaction requires a momentum cutoff, which is chosen at $500 \mathrm{MeV}$. In general, this $N N$ force induces relatively few high-momentum components in the many-body wave function. This is particularly true beyond the cutoff momentum, where the potential is not expected to apply. These three $N N$ interactions are phase-shift equivalent. In contrast, we also display a few results obtained with the Av4' reduction of Av18 [35]. This potential does not reproduce experimental $N N$ scattering phase shifts. In particular, it lacks tensor terms, but it is built to reproduce the deuteron binding energy. Av4' is therefore useful in assessing the importance of tensor correlations in the nuclear medium. A detailed analysis of the energy of nuclear and neutron matter with different versions of the Argonne potential has been recently reported in Ref. [36]. Furthermore, let us note that we have checked that the results obtained with Av6', the simplest Argonne interaction to include tensor terms, are very close to those provided by the fully realistic Av18. This is an indication of the importance of tensor terms in the physics at play discussed in the following.

In the following, we neglect the effect of three-body forces. Recent calculations indicate that the isospin asymmetry dependence of SRC is relatively insensitive to the presence of three-body interactions, at least around saturation density [23]. We note, however, that the usual BHF approach to include effective two-body forces obtained from three-body interactions by a simple density folding is not consistent with a diagrammatic expansion including antisymmetrized matrix elements [37]. Moreover, the density folding in isospin asymmetric matter has not been fully implemented [38]. Hence, a fully quantitative answer to this issue is still missing.

The present study complements, from a microscopic perspective, a series of recent analyses of the impact of highmomentum components on isovector properties [39-41]. Both schematic and purely microscopic calculations with different methods have identified the effect of SRCs in the kinetic component of the symmetry energy. In general, a correlated system has a larger kinetic energy than its uncorrelated counterpart. The redistribution of one-body strength underlying this increase, however, is different for different asymmetries. In particular, the asymmetry dependence is milder in the correlated case, which leads to a smaller kinetic symmetry energy. At low densities, this component can even become negative. Whether this reduction affects the observable bulk properties of neutron-star matter is still under discussion.

\section{FORMALISM}

A particularly relevant question, in view of recent advances $[25,26]$, is the dependence of SRCs with isospin asymmetry. In an arbitrarily isospin-polarized system, effects dominated by $n p$ pairs will have a different asymmetry dependence than those associated with same-isospin pairs. This can potentially have observable implications on single-particle properties. Here we look at the problem from the perspective of isospin asymmetric nuclear matter [42]. We will characterize isospin asymmetry using either the proton fraction, $x_{p}=\frac{\rho_{p}}{\rho_{n}+\rho_{p}}$, or the isospin-asymmetry parameter,

$$
\beta=\frac{\rho_{n}-\rho_{p}}{\rho_{n}+\rho_{p}}=1-2 x_{p} .
$$

In practice, our calculations are performed at finite temperature to avoid instabilities associated with pairing solutions $[11,43,44]$. While a proper treatment of pairing can be implemented [45], it is not relevant for the discussion of high-momentum components. In the following, all the results have been computed at a low temperature of $T=5 \mathrm{MeV}$. Although not shown here explicitly, we have checked that our conclusions are not seriously affected by working at finite temperature. We note that the high-momentum region is dominated by $N N$ correlations rather than thermal effects [11], and hence it is insensitive to temperature.

Correlations have a particularly clear manifestation in the single-particle momentum distribution [11],

$$
n_{\tau}(k)=\left\langle a_{\tau}^{\dagger}(k) a_{\tau}(k)\right\rangle
$$

where $a^{\dagger}(k)[a(k)]$ is the creation (destruction) operator of a single-particle excitation with momentum $k$ and isospin index $\tau=n, p$. The average, $\langle\cdot\rangle$, is taken over a complete thermal set of many-body states [12]. Our aim is to quantify how one-body high-momentum components evolve with density and isospin asymmetry. To access these density and asymmetry dependencies, we perform SCGF calculations at arbitrary densities and isospin asymmetries [44]. We provide here a concise explanation of the ladder SCGF method. A detailed description of the numerical techniques employed in its resolution can be found elsewhere $[44,46]$. 
The numerical simulations are obtained from a selfconsistent summation of ladder diagrams in the two-body sector which accounts consistently for SRC and tensor effects. This is achieved by means of an in-medium LippmannSchwinger equation, which can be schematically represented as

$$
\mathcal{T}_{\tau+\tau^{\prime}}=V_{\tau+\tau^{\prime}}+V_{\tau+\tau^{\prime}} G_{I I, \tau \tau^{\prime}}^{0} \mathcal{T}_{\tau+\tau^{\prime}}
$$

The sums $\tau+\tau^{\prime}$ stand for the three relevant channels for in-medium scattering in asymmetric matter, namely neutronneutron $(n n)$, proton-proton $(p p)$, and $n p$. The isospindependent $N N$ interaction, $V$, is summed to all orders to obtain a well-behaved in-medium effective interaction, or $\mathcal{T}$ matrix. We solve the Lippmann-Schwinger equation using matrix techniques with up to $J=4$ partial waves in all isospin channels.

In-medium scattering is mediated by a lowest-order twobody propagator, $G_{I I, \tau \tau^{\prime}}^{0}$, which accounts for both particleparticle and hole-hole correlations and incorporates off-shell effects completely. In asymmetric matter, the different isospin elements of this propagator are given by

$$
\begin{aligned}
& G_{I I, \tau \tau^{\prime}}^{0}\left(k, k^{\prime} ; \Omega\right) \\
& \quad=\int \frac{d \omega}{2 \pi} \frac{d \omega^{\prime}}{2 \pi} \frac{\mathcal{G}_{\tau}^{<}(k, \omega) \mathcal{G}_{\tau^{\prime}}^{<}\left(k^{\prime}, \omega^{\prime}\right)-\mathcal{G}_{\tau}^{>}(k, \omega) \mathcal{G}_{\tau^{\prime}}^{>}\left(k^{\prime}, \omega^{\prime}\right)}{\Omega-\omega-\omega^{\prime}+i \eta} .
\end{aligned}
$$

The two components of the single-particle propagator, $G_{\tau}^{<}$and $G_{\tau}^{>}$, are related by a Kubo-Martin-Schwinger relation [28]. They can also be linked to the single-particle spectral function, $\mathcal{A}_{\tau}$,

$$
\begin{aligned}
& \mathcal{G}_{\tau}^{<}(k, \omega)=f_{\tau}(\omega) \mathcal{A}_{\tau}(k, \omega), \\
& \mathcal{G}_{\tau}^{>}(k, \omega)=\left[1-f_{\tau}(\omega)\right] \mathcal{A}_{\tau}(k, \omega),
\end{aligned}
$$

using the Fermi-Dirac distribution of each species, $f_{\tau}(\omega)=$ $\left[1+e^{\frac{\omega-\mu_{\tau}}{T}}\right]^{-1}$. The chemical potential, $\mu_{\tau}$, is found from the integral of the momentum distribution, $n_{\tau}$. We normalize the distribution to unity,

$$
\int_{0}^{\infty} d k k^{2} n_{\tau}(k)=1
$$

which allows for clear comparisons with a finite system like the deuteron [8]. The momentum distribution is related to the single-particle propagator via a density-dependent factor:

$$
n_{\tau}(k)=\frac{2}{\pi^{3} \rho_{\tau}} \int_{-\infty}^{\infty} d \omega G_{\tau}^{<}(k, \omega)
$$

The combination of the two expressions, Eqs. (7) and (8), provides a nontrivial connection between the partial density, $\rho_{\tau}$, and the chemical potential, $\mu_{\tau}$.

Self-consistency is imposed all the way through in our calculations. The single-particle propagators that enter Eq. (4), for instance, are obtained from the $\mathcal{T}$ matrix itself. In the ladder approximation, this effective interaction defines the imaginary part of the (retarded) self-energy,

$$
\begin{aligned}
\operatorname{Im} \Sigma_{\tau}(k, \omega)= & \sum_{\tau^{\prime}} \int \frac{d^{3} k^{\prime}}{(2 \pi)^{3}} \int \frac{d \omega^{\prime}}{2 \pi}\left[f_{\tau^{\prime}}\left(\omega^{\prime}\right)+b_{\tau, \tau^{\prime}}\left(\omega+\omega^{\prime}\right)\right] \\
& \times\left\langle\boldsymbol{k} \boldsymbol{k}^{\prime}\left|\operatorname{Im} \mathcal{T}_{\tau+\tau^{\prime}}\left(\omega+\omega^{\prime}\right)\right| \boldsymbol{k} \boldsymbol{k}^{\prime}\right\rangle_{A} \mathcal{A}_{\tau^{\prime}}\left(k^{\prime}, \omega^{\prime}\right),
\end{aligned}
$$

which acquires different contributions from equal and unequal isospin partners. The difference arises from both the fermionic, $f_{\tau}(\omega)$, and the bosonic, $b_{\tau, \tau^{\prime}}(\Omega)=\left[e^{\frac{\Omega-\mu_{\tau}-\mu_{\tau^{\prime}}}{T}}-1\right]^{-1}$, phasespace factors. Furthermore, there is also an isospin dependence associated with the isospin-channel-dependent retarded $\mathcal{T}$ matrix, which is properly antisymmetrized in the relevant channels.

The dispersive contribution to the real part of the selfenergy can be obtained from a dispersion relation [12]. The generalized, instantaneous Hartree-Fock contribution,

$$
\Sigma_{\tau}^{\mathrm{HF}}(k)=\sum_{\tau^{\prime}} \int \frac{d^{3} k^{\prime}}{(2 \pi)^{3}} \int \frac{d \omega^{\prime}}{2 \pi}\left\langle\boldsymbol{k} \boldsymbol{k}^{\prime}\left|V_{\tau+\tau^{\prime}}\right| \boldsymbol{k} \boldsymbol{k}^{\prime}\right\rangle_{A} G_{\tau^{\prime}}^{<}\left(k^{\prime}, \omega^{\prime}\right),
$$

must be included as well. We consider up to $J=8$ partial waves in the calculation of this contribution, which is relevant for in-medium quasiparticle shifts. The solution of the Dyson equation generates the single-particle spectral function:

$$
\mathcal{A}_{\tau}(k, \omega)=\frac{-2 \operatorname{Im} \Sigma_{\tau}(k, \omega)}{\left[\omega-\frac{k^{2}}{2 m}-\operatorname{Re} \Sigma_{\tau}(k, \omega)\right]^{2}+\left[\operatorname{Im} \Sigma_{\tau}(k, \omega)\right]^{2}} .
$$

Feeding this spectral function into Eqs. (4)-(6), one obtains a self-consistency loop that treats all particles in the same manner, providing feedback to the different ingredients of the calculations. This goes beyond the scope of other approaches in providing an asymmetry-dependent spectral function and hence a fully correlated description of the microscopic properties of the system. In addition, macroscopic bulk properties can be accessed by means of sum rules and the Luttinger-Ward formalism $[11,41,44]$.

\section{DENSITY DEPENDENCE OF SHORT-RANGE CORRELATIONS}

In our previous work, we have investigated the asymmetry dependence of the (depleted) low-momentum components of the one-body momentum distribution [11]. In that case, because the dependence on momentum below the Fermi surface is particularly mild $[47,48]$, one can focus on the single, lowest available momentum $k=0$. In contrast, any attempt to quantify effects associated with high momenta will necessarily depend on the definition of such "high momenta." Here, we define high-momentum regions as ranging from $\sim 400$ to $\sim 850 \mathrm{MeV}$. At the lower end, this is far enough from the Fermi surface to avoid effects associated to its smoothing with 

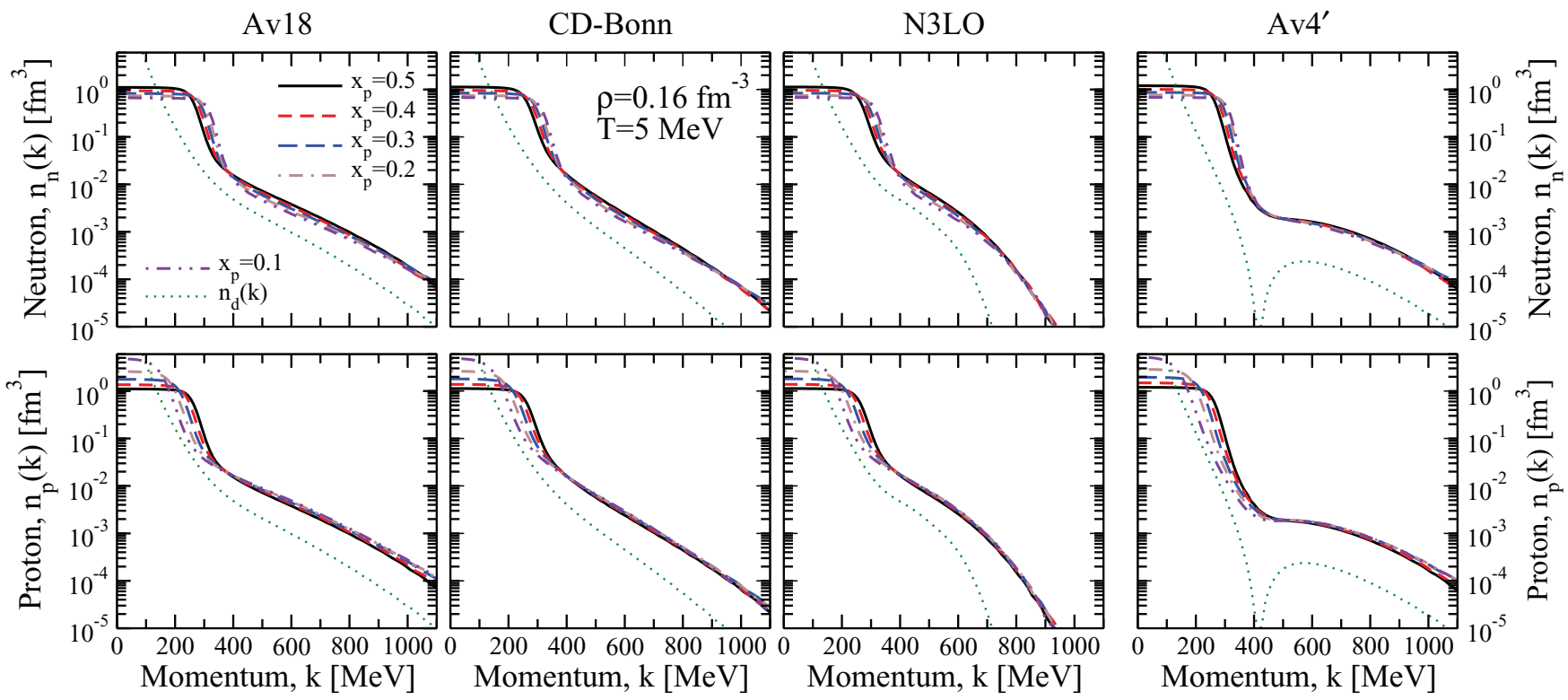

FIG. 1. (Color online) Momentum distribution for neutrons (top panels) and protons (bottom panels) in asymmetric nuclear matter. Different panels correspond to different $N N$ interactions, from left to right: Argonne v18, CD-Bonn, N3LO, and Av4'. Lines represent data obtained at different proton fractions. We also show, for comparison, the momentum distribution of the deuteron (dotted line) associated with each interaction.

temperature at different asymmetries. ${ }^{1}$ At the high end, this range represents the limit at which momentum distributions stop scaling, as we see next. Within high momenta, we also define a "tensor-dominated" momentum region, ranging from $k \sim 400 \mathrm{MeV}$ to $k \sim 550 \mathrm{MeV}$ (see below for details).

We start the discussion by showing in Fig. 1 the momentum distribution of neutrons (top panels) and protons (bottom panels) at different asymmetries for a fixed total density of $\rho=0.16 \mathrm{fm}^{-3}$ and a temperature of $T=5 \mathrm{MeV}$. The different columns represent results obtained with different $N N$ interactions. We focus our attention on the high-momentum region, well above the Fermi surface, and hence plot our results in a logarithmic scale. Overall, we note that the high-momentum components change very little when the isospin asymmetry is modified. This suggests that, when the momentum distribution is normalized to unity, highmomentum components are basically determined by the total density of the system.

We compare the results of asymmetric infinite matter to the corresponding deuteron momentum distribution, shown as the dotted line. Qualitatively, and as expected, the highmomentum components in the momentum distribution of nuclear matter are qualitatively similar to those of the corresponding deuterons. For the phase-shift equivalent interactions, we identify an area, in the momentum region 400-550 MeV, where the slopes of the two distributions are very much alike. In contrast, $\mathrm{Av} 4^{\prime}$ results (rightmost column), which lack tensor effects, show a node in the momentum

\footnotetext{
${ }^{1}$ This momentum is also close to point at which a pure $S$-wave deuteron would have a node in the momentum distribution; see right panels of Fig. 1.
}

distribution in this region. This confirms that the deuteron momentum distribution in this area is dominated by tensor effects, as observed in previous studies [14]. From now on, we call this the "tensor-dominated" region. We quantify the agreement between the two momentum distributions in the tensor-dominated region later.

Looking at the high-momentum components in more detail, we observe that the intrinsic momentum cutoff of N3LO shows up naturally in the momentum distribution (third column). The distribution displays a sharp decrease above $500 \mathrm{MeV}$, absent in the other $N N$ interactions. In particular, the deuteron momentum distribution data obtained in Ref. [49] is not reproduced for such a soft interaction (or for an interaction with no tensor terms, such as Av4'). One can debate whether such high-momentum components are physically motivated in $N N$ interactions [50,51], but their presence in the many-body wave function seems unquestionable. While the short-range part of the $N N$ interaction is not constrained by a fit to scattering data, we note that all experiments indicate that no sharp drop associated with a cutoff can be expected $[8,49]$. We therefore adopt the interpretation that the short-range part of relative $N N$ wave functions must be suppressed because the probability to find two intact nucleons at such distances must vanish on account of their intrinsic structure. Which $N N$ interaction is to be preferred can then be benchmarked by demanding an appropriate description of the modest $10 \%$ of nucleons with momenta not contained in the mean field, as measured, for instance, in Ref. [3]. Moreover, calculations with different short-range $N N$ forces provide a range of theoretical results. One can take this spread as a measure of the uncertainty in the short-range description of $N N$ interactions.

We identify a small decreasing (increasing) trend in the neutron (proton) high-momentum components as the system 


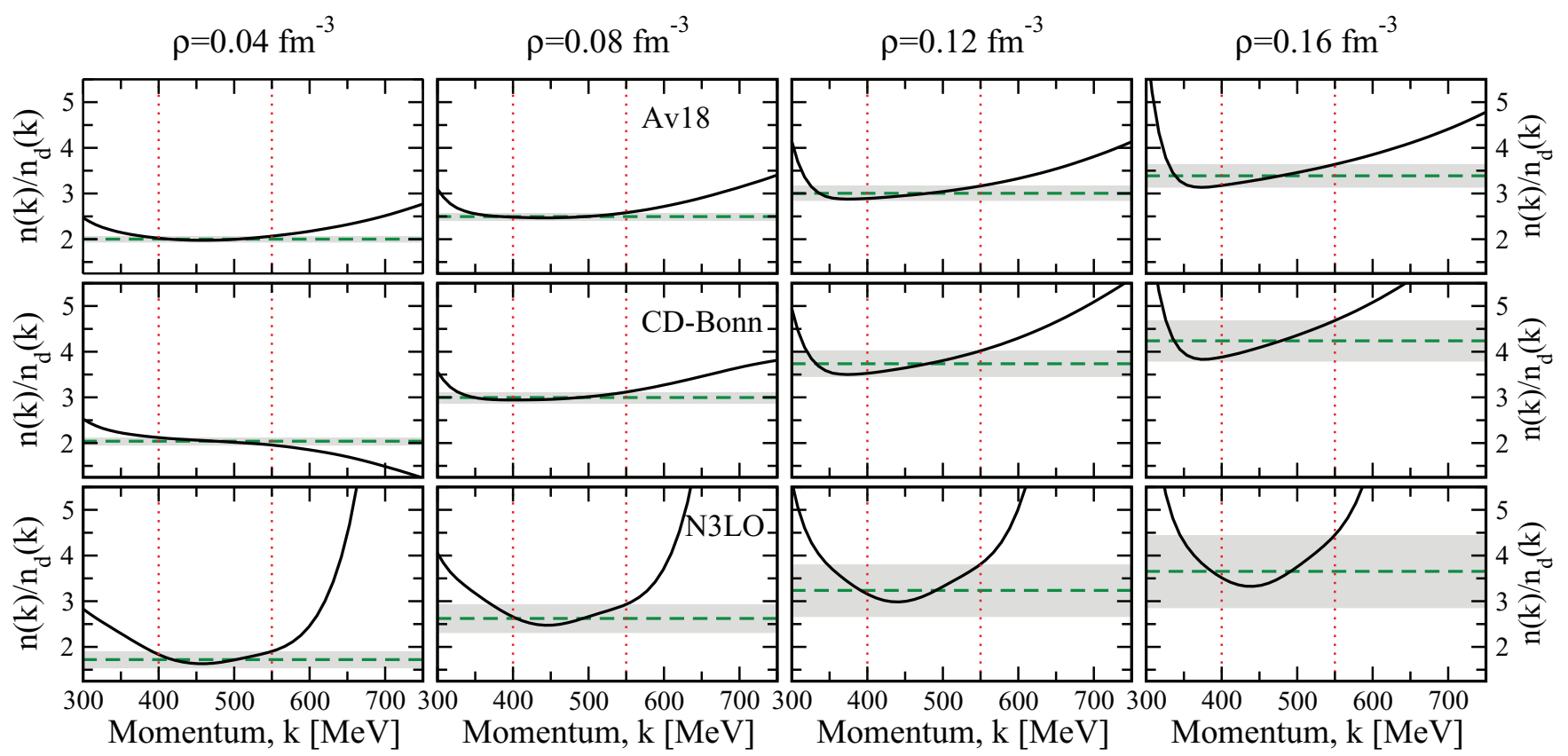

FIG. 2. (Color online) Ratio of the nucleon momentum distribution to the corresponding deuteron distribution at high momenta. Different columns correspond to different densities, from $\rho=0.04 \mathrm{fm}^{-3}$ (left column) to $\rho=0.16 \mathrm{fm}^{-3}$ (right column) in equidistant steps. Vertical panels show different $N N$ interactions: Argonne v18 (top row), CD-Bonn (middle row), and N3LO (bottom row) panels. The dashed horizontal line and gray region represent, respectively, the average and the error bar of the ratio in the tensor-dominated momentum region, depicted by vertical dotted lines.

becomes more neutron rich, which we analyze in more detail in Sec. IV. Above $600 \mathrm{MeV}$, the infinite matter results overshoot the deuteron ones. This happens for all interactions, including Av4'. We take this as an indication of the larger importance of SRCs in a dense system compared to the dilute deuteron bound state. In particular, the latter has a number density of $\rho \sim 2 /\left[4 \pi(5 / 3)^{3 / 2} r_{d}^{3} / 3\right] \sim 0.02 \mathrm{fm}^{-3}$, where $r_{d}$ is the deuteron charge radius. This density is well below saturation, where the results of Fig. 1 were obtained. We note that the SRC overshooting occurs well above the tensor-dominated region and hence the two regions should be distinguishable by applying momentum cuts.

One can quantify in more detail the similarity between $n_{\tau}(k)$ and $n_{d}(k)$, as well as the gradual overshoot at high momenta, by looking at the ratio of the two momentum distribution. This ratio is shown in continuous lines in Fig. 2 for different densities (columns) and three phase-shift equivalent interactions (rows). We draw three relevant observations from this figure.

First, there is a well-defined region in momentum where both distributions have similar momentum dependencies at subsaturation densities. Qualitatively, one can take this plateau region as an indication of the dominance of tensor correlations. The latter are of a similar nature in the deuteron and in the medium and hence lead to equivalent momentum dependencies in the distribution. There are arguments in favor of such similarity, from either hard-scattering physics [9] or factorization in the operator-product expansion [52].

Second, the ratio of the two distributions increases with density. This is most clearly seen in the tensor-dominated region, suggesting an increase in importance of tensorlike correlations with density. Because the relevant densities of nuclei are fairly similar to those of the deuteron, one should expect to see similar plateaus in finite systems [49]. Also, dense systems should have larger plateaus compared to dilute ones.

Third, the plateaus become less pronounced as the density increases. This suggests that the overshooting associated to SRC occurs at lower momenta with increasing $\rho$. One expects the nuclear medium to induce stronger correlations and hence lead to larger high-momentum tails as the density increases. This is particularly clear for N3LO (right panels), where the effect is enhanced by the strong falloff of $n_{d}(k)$ above the cutoff momentum. Overall, this agrees with the idea that mediuminduced SRC correlations become more relevant than tensorbased ones in the high-density region. The dominance of SRCs in the high-density region could have implications for neutronstar matter [24].

Different measures have been proposed to quantify the importance of tensor correlations. The ratio of the momentum distribution of a given nuclear system to that of the deuteron is one of such quantities. We refer to this measure as $a_{2}$, following Ref. [7]. ${ }^{2}$ More specifically, we define $a_{2}$ as the integrated average of the ratio in the tensor-dominated region:

$$
a_{2}=\left\langle\frac{n(k)}{n_{d}(k)}\right\rangle_{k=400-550 \mathrm{MeV}} .
$$

\footnotetext{
${ }^{2}$ Note that the same notation, namely $a_{2}$, has also been used to define other quantities such as cross-section ratios $[16,18]$.
} 
This is related, but not necessarily equal, to the probability of a single nucleon to be part of a SRC pair. Specifically, $a_{2}$ measures how many high-momentum nucleons there are in the distribution with respect to those in the deuteron. The horizontal dashed lines in Fig. 2 correspond to the values of the averaged $a_{2}$ for the different $N N$ interactions and densities. Our SCGF calculations suggest values of $a_{2}$ between $\sim 1$, at low densities, and $\sim 5$, at large densities. The node in the deuteron momentum distribution for $\mathrm{Av} 4^{\prime}$ would lead to divergent values of $a_{2}$. This demonstrates once again the relevance of tensor-induced correlations in this momentum region. We note that changing the average integration region by $\pm 50 \mathrm{MeV}$ gives quantitatively similar results.

For all three $N N$ interactions, the value of $a_{2}$ increases with density. The density dependence, however, is sensitive to the $N N$ interaction under consideration. As already mentioned, the plateau in the ratio of momentum distributions also becomes less and less prominent as the density increases. A clear minimum is, however, present in all cases, which suggests that one can still characterize the ratio in the tensor-dominated region using an average as in Eq. (12). To take into account any potential errors in the separation between tensor- and short-range-dominated regions, we have assigned a conservative uncertainty to $a_{2}$. This is represented in Fig. 2 by the gray bands, which have been obtained as the maximum deviation between the average and the function in the tensor-dominated momentum range. The shaded bands extend to momenta below the tensor-dominated region, cover the minimum in the ratio at all densities, and are wide enough to contain the numerical uncertainties associated with the solution of the SCGF equations. In general, we find that the error in $a_{2}$ increases with density. We still obtain, however, a meaningful increasing trend as a function of density for all $N N$ interactions, which we proceed to explore further.

We show in Fig. 3 the density dependence of $a_{2}$ obtained for the three relevant $N N$ forces. We note that, in all three cases, $a_{2}$ increases with density, saturating at high densities. We find modest differences between the potentials, which

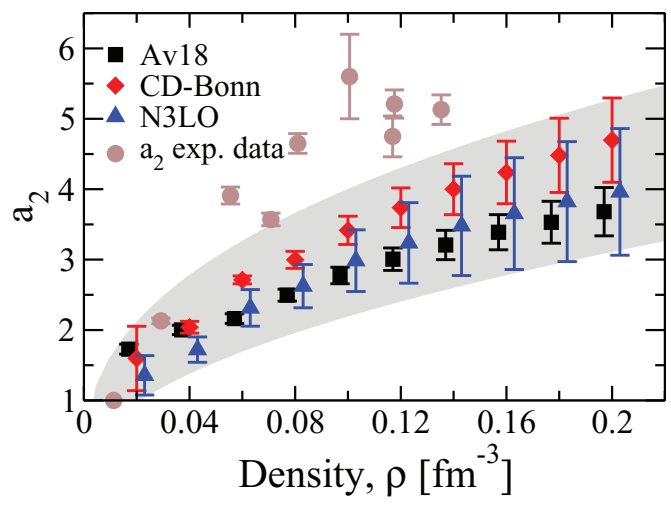

FIG. 3. (Color online) Density dependence of $a_{2}$, as defined in Eq. (12). We show values obtained for three $N N$ interactions, with a small horizontal offset for display purposes. Experimental $a_{2}$ values from Ref. [16] are plotted as a function of a rescaled nuclear density (see text for details). The shaded region corresponds to power laws with exponents $0.4-0.5$. at half saturation fall in the range $a_{2} \sim 2-2.7$. Somewhat surprisingly, CD-Bonn seems to provide the largest $a_{2}$. In other words, compared to the respective deuteron, CD-Bonn is a harder interaction than Av18. We have not found a simple quantitative explanation for this unforeseen result.

As already mentioned, the error bars increase with density for all three interactions, particularly for N3LO, where SRCs are hardly present. A two-parameter fit of the type $a_{2}=b_{1} \rho^{b_{2}}$ provides a range of values $b_{1}=7-10$ and $b_{2}=0.4-0.5$. This yields the range depicted in the gray region in Fig. 3, which contains all data points and their error bars.

We compare our values of $a_{2}$ to those extracted from a recent analysis of experimental data in Ref. [16]. Each experimental point in Fig. 3 corresponds to a different isotope, between the deuteron and gold, where experimental $\left(e, e^{\prime} p\right)$ data is available. These data have been reanalyzed recently in the context of the connection between the EMC effect and SRC [15,17]. Following this analysis, we have determined the number densities of the different isotopes by assuming a spherical liquid drop with radius equal to the charge radius of Ref. [53]. To this density, we have also applied a $\frac{A-1}{A}$ correction to account for the excess density seen by the knocked out nucleon, which propagates through a less dense environment. This should correspond to the so-called "scaled nuclear density" of Ref. [16]. We note that at low densities (deuteron and triton, essentially), the experimental data agree well with our numerical estimates of $a_{2}$. At moderate and large densities, however, the experimental data are well above our results. Nevertheless, the data of Ref. [16] are obtained as a ratio of deep inelastic cross sections rather than as a ratio of momentum distributions. The connection between the observables and the momentum distributions is not free of uncertainties. Let us stress, for instance, that our definition of $a_{2}$ does not account for the center-of-mass spreading of the $n p$ pair, a correction that can either reduce [16] or increase [18] the values of $a_{2}$ according to different theoretical models. A rescaling of the density or the theoretical values by a factor of 2 would lead to a good overall agreement between data and experiments.

\section{ISOSPIN-ASYMMETRY DEPENDENCE OF SHORT-RANGE CORRELATIONS}

Our results indicate a qualitative resemblance of the one-body high-momentum components of the deuteron and symmetric nuclear matter. We now want to investigate whether a similar resemblance is found in isospin-asymmetric matter. Our final aim is to quantify how much tensor-dominated correlations change with isospin asymmetry. We use the ratios of the calculated momentum distributions and the deuteron distribution as tools to assess the isospin dependence of tensorlike correlations. We show the ratio, for a fixed density of $\rho=0.08 \mathrm{fm}^{-3}$ and different isospin asymmetries, in the top panels of Fig. 4. We have chosen this relatively low density because in the symmetric case it still shows a clear scaling. We provide results for the three realistic phase-shift equivalent potentials.

The ratio of isospin-dependent momentum distributions reveals a noticeable dependence with isospin asymmetry 


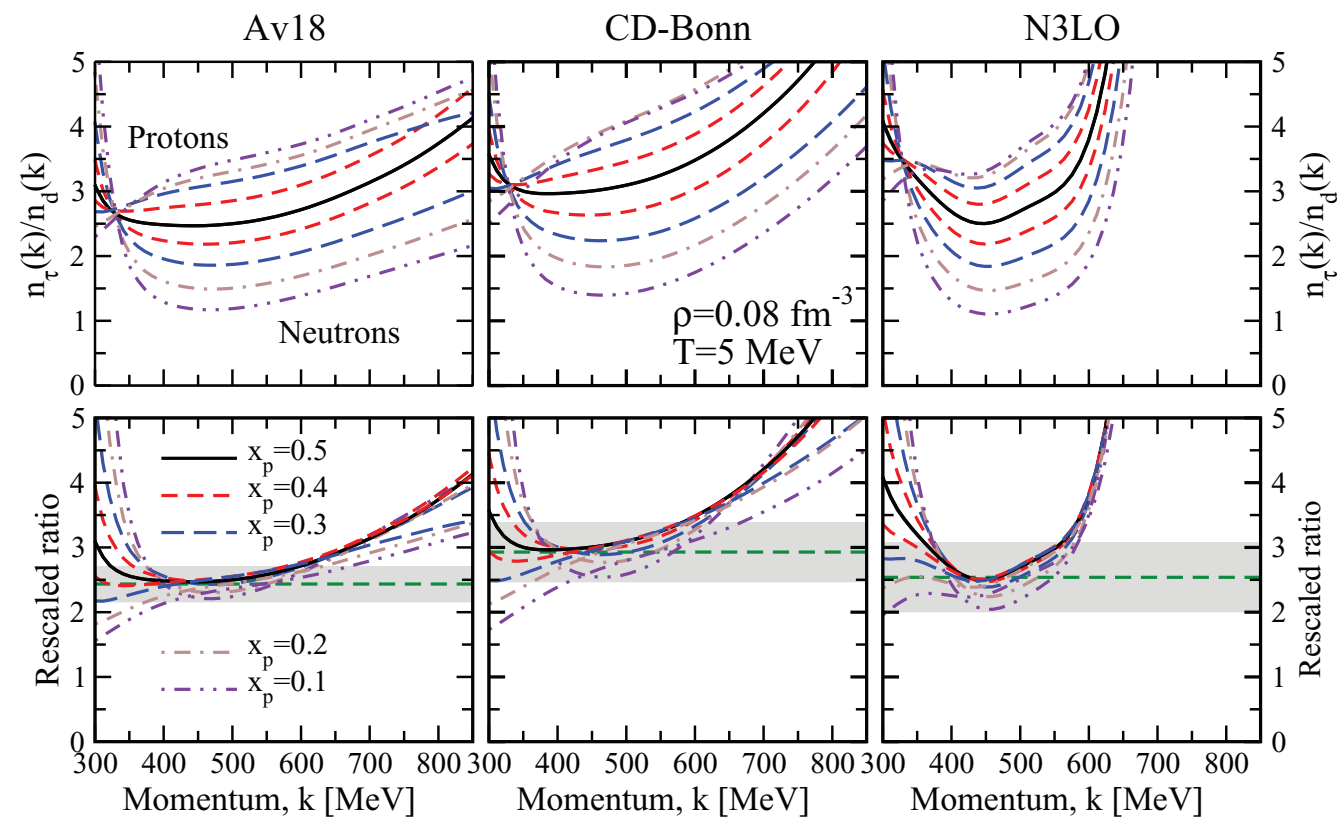

FIG. 4. (Color online) (Top panels) Ratio of the neutron and proton momentum distribution to the corresponding deuteron distribution at high momenta. Different line styles correspond to different isospin asymmetries. The three different panels correspond to thee $N N$ interactions, from left to right: Argonne v18, CD-Bonn, and N3LO. (Bottom panels) Rescaled neutron and proton momentum distributions, as discussed in the text. After rescaling, all results fall in a narrow band. Gray bands are an error band associated to the maximum deviation in the tensor-dominated region.

that was not apparent in Fig. 1. We find that the neutron distribution becomes less populated as the system becomes more neutron rich. Conversely, we observe an increasing trend for the proton momentum distribution. Taking this population as a measure of correlations, one can say that the minority species is more correlated. This agrees with the conclusions we drew when investigating the depletion of low-momentum, isospin-asymmetric components [11].

For all but the highest asymmetries in the proton distributions, the ratios evolve linearly with isospin asymmetry. We justify this linear dependence in the Appendix, with a derivation based on the DFG formalism in the high-momentum limit. This indicates that the asymmetry dependence of the momentum distribution should be given by

$$
n_{\tau}(k ; \rho, \beta)=[1 \pm \gamma(\rho) \beta] \times n_{\tau}(k ; \rho, 0),
$$

where $\gamma(\rho)$ is a dimensionless, density-dependent parameter that quantifies the isovector strength of the effective interaction and $\beta$ is the isospin asymmetry as defined in Eq. (1). More specifically, $\gamma$ should be associated with the isovector dependence of the effective interaction in the tensor channel. The latter parameter is negative, and therefore the plus (minus) sign corresponds to neutrons (protons). We have tested the validity of this asymmetry dependence for our numerical data at different densities and for a wide range of momenta. In particular, the asymmetry dependence holds very well in the whole tensor-dominated, $k=400-550 \mathrm{MeV}$ range and for all asymmetries. Factorization in an isospin asymmetric medium can presumably lead to a more quantitative justification of this linear dependence with asymmetry [52].
The bottom panels of Fig. 4 show rescaled ratios of the asymmetric matter and the deuteron momentum distributions. The rescaling is obtained by dividing the asymmetrydependent momentum distributions by $[1 \pm \gamma(\rho) \beta]$. The optimal $\gamma$ parameter is fitted at each density. In practice, we minimize at each asymmetry the difference between the rescaled ratio and that of the symmetric case. We then average over asymmetries to find an optimal $\gamma$ for both neutrons and protons at a given density. We provide error bars for this quantity in a similar fashion to the analysis for $a_{2}$, finding the maximum deviations between the data and the averages. In principle, this procedure could be followed independently for both neutrons and protons, thus deriving an isospin-dependent $\gamma$. Yet, we find a good agreement between the $\gamma$ parameters obtained independently. We take this as a confirmation of the quality of our proposed linear asymmetry dependence. We note that, after rescaling at a given density, the residual dependence on asymmetry in the tensor-dominated momentum range is effectively removed. This is a rather general property, occurring at densities as high as $\rho \sim 0.24 \mathrm{fm}^{-3}$.

In contrast, Sargsian [26] has suggested that a scaling of the type $n_{\tau}(k ; \rho, \beta) \sim[1 \pm \beta]^{-\gamma} n_{\tau}(k ; \rho, 0)$ with $\gamma \sim 1$ should be valid. We find that $\gamma=1$ does not provide a qualitatively good scaling of our data. In fact, it is not easy to find a power that reproduces the ratios at all asymmetries. We note, however, that the power-law scaling reduces to Eq. (13) in the small isospin-asymmetry limit. In particular, nearly symmetric systems like stable finite nuclei might be well reproduced by a power law in asymmetry. Further work to validate this asymmetry dependence in finite nuclei would have to be explored with a different methodology $[18,27,54]$. We note, 


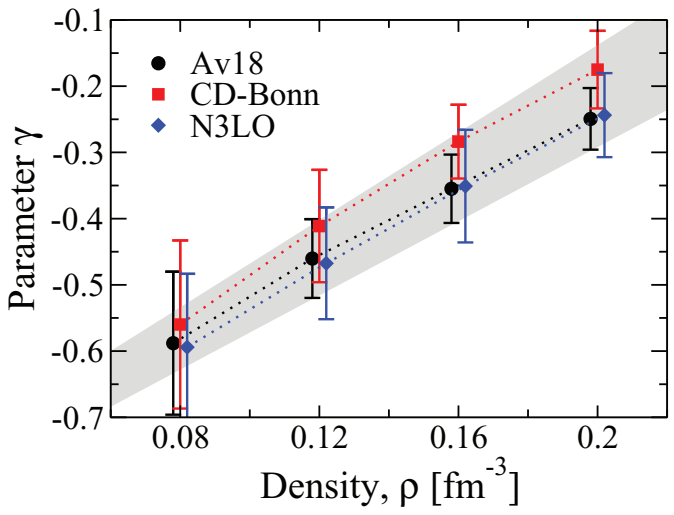

FIG. 5. (Color online) Density dependence of the $\gamma$ parameter for the three phase-shift equivalent interactions considered in this work. Data for each $N N$ force have a small horizontal offset for display purposes. Error bars have been calculated as differences between the optimal neutron and proton $\gamma$ parameters. The shaded region corresponds to linear fits with slopes $2.8-3.3 \mathrm{fm}^{3}$.

however, that extrapolations to neutron-star matter require the explicit linear dependence of Eq. (13).

We present in Fig. 5 the results of the averaged neutron and proton $\gamma$ computed at different densities. The error bars represent the maximum differences between the optimal fits of the neutron and proton distributions at different asymmetries. Again, this is a rather conservative estimate, generally larger than the fitting error of the individual $\gamma_{\tau}$. In practice, the parameters are very close to each other for different phase-shift equivalent interactions, which suggests that this scaling is universal and independent of the short-range components. In other words, the isovector dependence of correlations in the tensor-dominated region is very much restricted by phase-shift equivalence. Moreover, the density dependence of $\gamma$ is similar for different interactions. In the range of densities considered, and with the present data, we find that the density dependence is almost linear. The gray band in Fig. 5 corresponds to a range of regressions with linear slopes $2.8-3.3 \mathrm{fm}^{3}$ and origins between -0.80 and -0.85 .

Overall, our results suggest that the momentum distribution in asymmetric matter within the momentum range $400-550 \mathrm{MeV}$ is proportional to the deuteron momentum distribution. Given our numerical SCGF data, we propose the following density and asymmetry dependence of $a_{2}$ :

$$
a_{2, \tau}(\rho, \beta)=b_{1}[1 \pm \gamma(\rho) \beta] \rho^{b_{2}} .
$$

We have confirmed the density dependence of the symmetric matter $a_{2}$ using our asymmetric momentum distribution data. These are shown in a horizontal dashed line in the bottom panels of Fig. 4. The agreement with the $\beta=0$ case is good. The error bars are somewhat larger, owing to the additional uncertainties in the isospin-dependent nuclear-matter case.

Up to this point, we have used our results to highlight a qualitative scaling of the high-momentum components with the local density of the nuclear medium. Further, one can discuss whether the momentum dependence itself is sensitive to the isospin asymmetry. The ratio of momentum distributions, $n_{n}(k) / n_{p}(k)$, provides useful information on this issue. The top panels of Fig. 6 show the ratio at a fixed total density for different isospin asymmetries in the tensor-dominated region. For symmetric systems (solid line), the two distributions are the same at all momenta, as expected. When asymmetry is switched on $\left(x_{p}=0.4\right)$, one observes a well-defined plateau in the whole high-momentum region, even beyond $k \sim 600 \mathrm{MeV}$. The ratio is smaller than one, indicating that neutrons populate less the high-momentum components as compared to protons, as we have already seen. This might be relevant for heavy nuclei, which have bulk asymmetries of this order. Both momentum distributions have similar high-momentum dependence, but with proton populations about $15 \%$ larger than the ones for neutrons [26]. At the largest asymmetries explored here, similar to those found in neutron stars, this percentage increases to $\sim 40 \%$.

At large asymmetries, the plateau is well defined for Av18 and CD-Bonn, which have a strong short-range core. The scaling is less defined in $\mathrm{N} 3 \mathrm{LO}$, owing to the decay at momenta above $500 \mathrm{MeV}$. In all cases, the departure from 1 is stronger when asymmetry increases. The plateau in the momentum dependence also worsens as asymmetry increases. Again, this suggests a picture in which the asymmetry dependence of both momentum distributions is attributed to the tensor effects, with protons populating more high-momentum components as their fraction decreases. We note that the crossing around $400 \mathrm{MeV}$ is the same for all $N N$ interactions.

A further quantitative test of Eq. (13) can be obtained by rescaling the independent neutron and proton momentum distributions with the same $\gamma$ and then taking their ratio. If all the isospin asymmetry is correctly included in the scaling prefactor, all lines at different asymmetries should fall within a narrow band. The bottom panels of Fig. 6 show the accuracy of this rescaling procedure. We find that, after rescaling, all the asymmetry dependence is removed. The ratios at different asymmetries fall within $10 \%$ of each other in the tensor-dominated region. Above $k \sim 600 \mathrm{MeV}$, where SRCs dominate, we find differences for the larger asymmetry cases. This is particularly clear for N3LO. All in all, the figure suggests that the information on the momentum distribution in one asymmetric system might be enough to extrapolate to other asymmetric systems. A linear extrapolation using the universal $\gamma$ parameter is enough for these purposes. This procedure should work well for the tensor-dominated region, with larger uncertainties for the short-range-dominated region.

\section{ASYMMETRY DEPENDENCE OF INTEGRATED STRENGTH}

So far, we have paid particular attention to the momentum dependence of the distribution. In some cases, however, one might be interested in looking at more global, or integrated, properties [26]. We thus now proceed to analyze the integrated one-body strength in the high-momentum region. This provides an insight into the mechanisms that populate highmomentum components and hence their importance beyond the independent-particle model. We quantify the population 

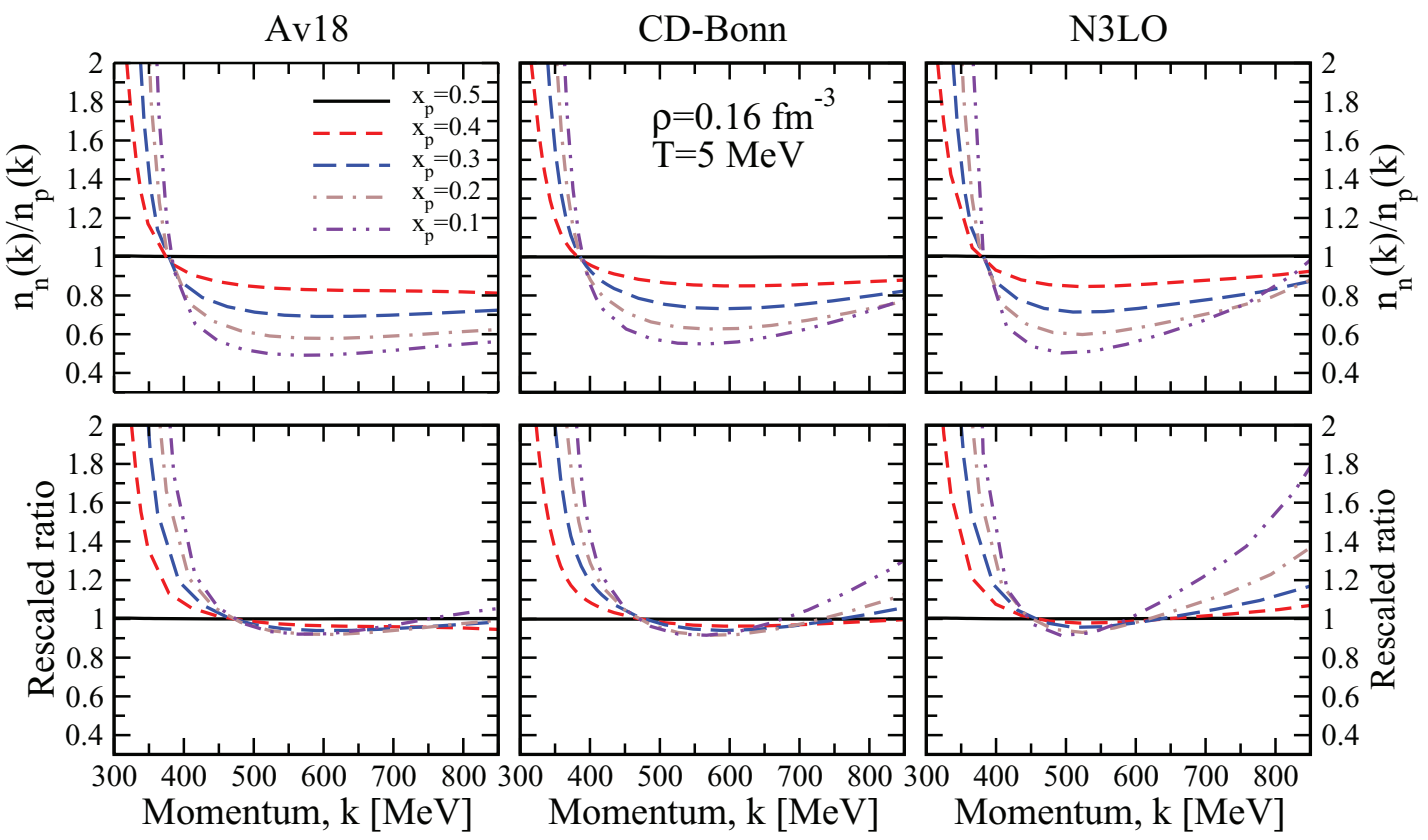

FIG. 6. (Color online) (Top panels) Ratio of the neutron to the proton momentum distribution for different proton fractions. (Bottom panels) Rescaled ratio of momentum distributions (see text for details). Different columns correspond to different $N N$ interactions: Argonne v18 (left panels), CD-Bonn (middle panels), and N3LO (right panels).

using the integrated strength,

$$
\phi_{2}\left(k_{i}, k_{f}\right)=\frac{1}{\pi^{2} \rho_{\tau}} \int_{k_{i}}^{k_{f}} d k k^{2} n_{\tau}(k),
$$

in three different momentum regions. The low-momentum region, from $k_{i}=0$ to $k_{f}=400 \mathrm{MeV}$, includes depletion effects as well as the shifts in the Fermi momenta. In a free Fermi gas picture at $\rho=0.16 \mathrm{fm}^{-3}$ and $T=5 \mathrm{MeV}$, the thermal depletion in this region is very small at all asymmetries, in accordance with the Pauli principle. The top panels of Fig. 7 show the asymmetry dependence of this region for both neutrons (circles) and protons (squares). As expected, we find a depletion of states at low momenta, with a departure with respect to 1 . The observed isovector splitting is therefore necessarily induced by beyond-mean-field correlations, with neutrons becoming more and more populated (that is, less correlated) as the system becomes more neutron-rich. This picture is in accordance with our previous results [11]. Av4' data is less affected by isospin asymmetry compared to the data of other phase-shift equivalent potentials. We take this as yet another indication that tensor correlations are relevant for the isovector dependence of the momentum distribution.

The middle panels of Fig. 7 represent the tensor-dominated region of interest for our study. In symmetric matter for phase-shift equivalent $N N$ forces, this region contains between $6 \%$ and $8 \%$ of the single-particle strength. As asymmetry increases, however, high-momentum protons become more and more prominent. This is necessarily related to the decrease in the low-momentum region, because the overall strength is normalized. For a hard interaction such as Av18, and for fractions of $x_{p} \sim 0.1$, typical of neutron-star matter, up to $10 \%$ of the proton strength is in this high-momentum region. Conversely, neutrons populate less and less of the region, down to $4 \%-5 \%$, as the system becomes more neutron-rich. As we have already seen, the quantitative details depend on the short-range and tensor structure of the $N N$ force under consideration. The qualitative picture, however, is extremely robust. Protons become more prominent at high momenta as their concentration decreases. In this sense, one can say that protons are more correlated in a proton-poor environment. The limit $x_{p} \rightarrow 0$ would provide further insight, of relevance for polaron physics [55].

The bottom panels of Fig. 7 illustrate the remaining strength in the very high-momentum region, above $850 \mathrm{MeV}$. Only interactions with a very strong short-range core, such as Av18, are able to promote particles to this region, with typical populations of $\sim 1 \%$. We note that this happens also for Av4' and for Av6' (not shown here for brevity), which indicates that this region is insensitive to the tensor structure of the force. The neutron-proton splitting here also becomes very small, if at all relevant.

Finally, we want to assess the importance of highmomentum components in a bulk quantity that depends on isospin asymmetry. The kinetic energy is particularly sensitive to such components [41], as it is an integral involving $n_{\tau}(k)$. We provide in Table I the values of the neutron (proton) kinetic energy per neutron (proton) as a function of $x_{p}$. In symmetric matter, one observes the expected ordering, with the stronger short-range core potential, Av18, providing the largest values. Conversely, N3LO, the softest interaction, produces the lowest value.

As the isospin asymmetry increases, one observes a common trend: neutron kinetic energies increase, whereas proton energies decrease. This indicates that, even though protons dominate the high-momentum region, the growing contribution of neutrons in the low-momentum area still dominates the 


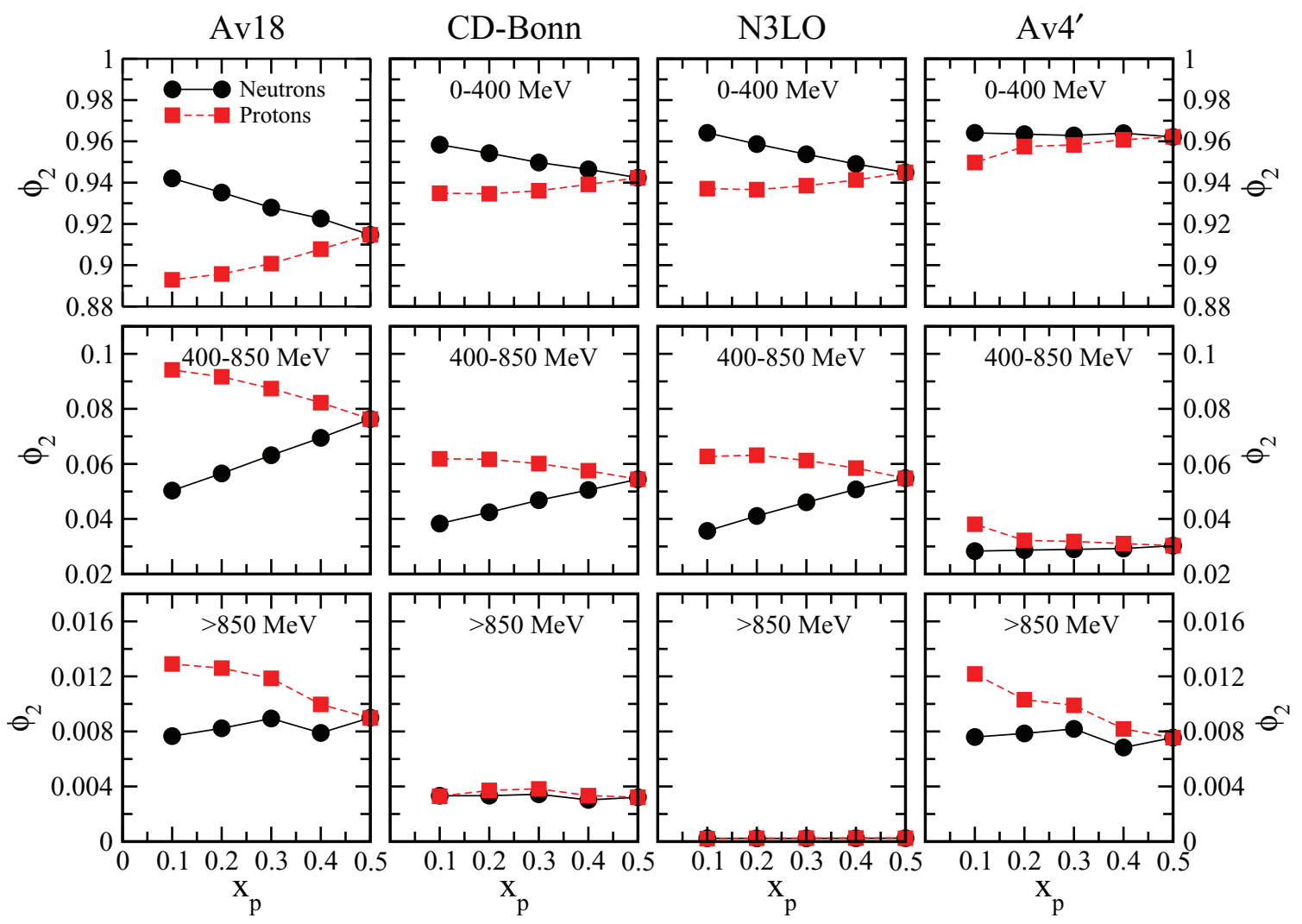

FIG. 7. (Color online) Integrated single-particle strength for neutrons (circles) and protons (squares) in three momentum regions: $k=$ $0-400 \mathrm{MeV}$ (top panels), $k=400-850 \mathrm{MeV}$ (middle panels), and $k>850 \mathrm{MeV}$ (bottom panels). The four columns correspond to different $N N$ interactions. Note the different vertical scales of the different rows.

kinetic energy. The isovector splitting of kinetic energies is fairly symmetric and almost linear, except for protons in the most asymmetric region, which we take as an indication of the appearance of low-momentum thermal effects. Curiously, even though Av18 provides the largest isovector splittings in the strength, it provides the lowest splitting in kinetic energies. This has to do with the redistribution of spectral strength as a function of momentum, which is weighted differently in the one-body strength than in the kinetic energy.

We also note that the total kinetic energy is the weighted average of the proton and neutron components. Consequently, the neutron kinetic energy dominates the result as $x_{p}$ decreases. The linear dependence of the individual energy fractions

TABLE I. Neutron (proton) kinetic energy per neutron (proton) for different proton fractions. All energies are in $\mathrm{MeV}$ and results for three $N N$ interactions are shown.

\begin{tabular}{lcccccc}
\hline \hline & $x_{p}$ & 0.5 & 0.4 & 0.3 & 0.2 & 0.1 \\
\hline Av18 & $K_{n}$ & 41.8 & 42.3 & 44.2 & 44.8 & 45.6 \\
& $K_{p}$ & 41.8 & 41.0 & 40.5 & 39.0 & 36.5 \\
CD-Bonn & $K_{n}$ & 35.0 & 36.5 & 38.4 & 39.8 & 41.2 \\
& $K_{p}$ & 35.0 & 33.2 & 31.4 & 28.7 & 25.3 \\
N3LO & $K_{n}$ & 32.9 & 34.6 & 36.1 & 37.5 & 39.0 \\
& $K_{p}$ & 32.9 & 31.1 & 28.8 & 26.2 & 22.7 \\
\hline \hline
\end{tabular}

suggests that the total kinetic energy will be quadratic in isospin asymmetry. Overall, however, the isospin dependence is rather mild, so that the kinetic component of the symmetry energy decreases with respect to the free Fermi gas case [41]. This is the only effect that we are able to identify as correlation-driven. Our results do not seem to suggest that the tensor-dominated region is responsible for any dramatic changes in total energy, at least around saturation density.

\section{CONCLUSIONS}

We have reviewed the density and isospin-asymmetry dependence of the high-momentum components of the onebody momentum distribution. The latter were obtained at arbitrary asymmetries and different densities by means of SCGF ladder calculations. These are particularly well-suited to study correlated momentum distributions, as they provide fully fragmented single-particle propagators. To avoid the arbitrariness associated with the short-range or tensor structure of the $N N$ potential, we have performed calculations with a variety of interactions. These include phase-shift equivalent forces with strong and soft short-range cores and with and without tensor terms.

Our analysis shows that if the momentum distribution is normalized to one, the high-momentum components of each $N N$ force become almost universal and asymmetry 
independent. Comparing with the associated deuteron of each interaction, we see that in-medium SRCs tend to provide larger high-momentum components than the deuteron above $\sim 600 \mathrm{MeV}$. By taking a ratio of $n(k)$ to $n_{d}(k)$, however, we have been able to identify a region of momentum, between 400 and $550 \mathrm{MeV}$, where the two distributions have similar dependencies. The comparison with a tensor-free interaction indicates that this region is dominated to a large extent by the tensor components of the $N N$ interactions. The quality of the scaling of the two distributions decreases substantially with density because of the increasing importance of SRC.

The tensor-dominated region of $k \sim 400-550 \mathrm{MeV}$ is of interest, as it is expected that the momentum distribution here is rather universal [7]. Our calculations have demonstrated that the ratio in this region can be characterized by a single parameter, which we refer to as $a_{2}$. This is related to the probability of finding short-range-correlated nucleons in the medium compared to the deuteron. The latter increases with density and is somewhat dependent on the underlying $N N$ interaction. We find that CD-Bonn provides the larger values of $a_{2}$, followed by N3LO and Av18. The uncertainty in this quantity increases with density as well, but our predictions still fail to reproduce experimental values. This could either be attributable to an issue with pair counting or with interpretation of the data. We have parametrized its density dependence in terms of a power law of exponent $\sim 0.4-0.5$. Interactions without tensor components, however, produce divergent results for $a_{2}$.

We observe that isospin asymmetry shifts the in-medium momentum distributions to higher (lower) values for protons (neutrons). This indicates a prevalence of high-momentum components of the minority species as the asymmetry increases, which confirms previous results. Using a DFG model as a guiding principle, we have verified that the residual asymmetry dependence in the high-momentum region is almost linear with asymmetry. The parameter that drives this asymmetry dependence is negative, but approaches zero as the density increases. Moreover, we find that this parameter is independent of the $N N$ interactions. This suggests that the isovector dependence of correlations is rather model independent, in agreement with previous results [11].

Finally, we have turned our attention to integrated quantities, which also show a dependence on correlations and asymmetry. Dividing the momentum distribution in different regions, we find that low-momentum neutrons are less correlated in neutron-rich matter. Conversely, the tensor- dominated region is predominantly populated by protons as the system becomes more asymmetric. The isovector splittings are qualitatively similar for all interactions, except for those missing tensor terms. Regarding the kinetic energy of each component, we have found an asymmetry dependence around saturation. However, the redistribution of strength is such that the neutron kinetic energy in neutron-rich systems is larger than in symmetric ones. In other words, even though neutrons populate more the low-momentum region as asymmetry increases, this change is not as dramatic as that of the neutron fraction itself. We note that this asymmetry dependence has implications for symmetry energy studies [41].

Two-body observables can provide further insight into the interplay of high-momentum components, tensor correlations, and isospin asymmetry $[18,27,30]$. These are beyond the scope of the present work, but they can be generated in the SCGF framework. Access to such properties will provide further, much-needed quantitative understanding of SRCs in infinite and arbitrarily isospin-polarized nuclear systems.

\section{ACKNOWLEDGMENTS}

This work is partly supported by by the Consolider Ingenio 2010 Programme CPAN CSD2007-00042, Grant No. FIS2011-24154 from MICINN (Spain), and Grant No. 2009GR-1289 from Generalitat de Catalunya (Spain); by STFC, through Grants No. ST/I005528/1 and No. ST/J000051; and by the US National Science Foundation under Grants No. PHY-0968941 and No. PHY-1304242.

\section{APPENDIX: DILUTE FERMI GAS}

The DFG model provides a useful perspective into the microscopic properties of correlated homogeneous fermionic systems. This model is based on the low-density Lee-Yang expansion for strongly interacting systems. Essentially, one recasts the many-body problem in terms of an effective, scattering matrix and uses it in a perturbative expansion similar to Ref. [56]. The low-energy scattering is characterized by a scattering length, $a$. Expressions for the momentum distribution of unpolarized systems were derived up to order $\left(k_{F} a\right)^{2}$ by Sartor and Mahaux [29]. We are not aware of any calculations for polarized systems using this model.

For an unpolarized system of fermions with mass $m$, the momentum distribution in the region above the Fermi surface, $k>k_{F}$, is given by $[28,29]$

$$
n(k)=\frac{v(v-1)}{\left(2 \pi^{2}\right)^{3}} \frac{a^{2}}{\rho m^{2}} \int_{k_{1}>k_{F}} d^{3} \mathbf{k}_{\mathbf{1}} \int_{k_{2}<k_{F}} d^{3} \mathbf{k}_{\mathbf{2}} \int_{k_{3}<k_{F}} d^{3} \mathbf{k}_{\mathbf{3}} \frac{\delta^{(3)}\left(\mathbf{k}+\mathbf{k}_{\mathbf{1}}-\mathbf{k}_{\mathbf{2}}-\mathbf{k}_{\mathbf{3}}\right)}{\left[\frac{k^{2}+k_{1}^{2}-k_{2}^{2}-k_{3}^{2}}{2 m}\right]^{2}},
$$

where we have introduced the degeneracy, $v=4(2)$ for symmetric (neutron) matter. We only consider the $k \gg 1$ limit of this model, which is particularly easy to compute and provides an indication of the asymmetry dependence. For an unpolarized system, the limit can be found by noticing that the external momentum becomes larger than any other scale in the system. Pulling it out of the integral, one finds

$$
n(k) \rightarrow n^{\gg}(k)=\frac{v(v-1)}{\left(2 \pi^{2}\right)^{3}} \frac{a^{2}}{\rho k^{4}} \int_{k_{2}<k_{F}} d^{3} \mathbf{k}_{\mathbf{2}} \int_{k_{3}<k_{F}} d^{3} \mathbf{k}_{\mathbf{3}}=\frac{v-1}{v} \frac{8 a^{2} \rho}{k^{4}} .
$$

Similar power-law behaviors at high momenta, albeit with different exponents, are also obtained in hard scattering calculations [9] or in the electron gas [52]. 
Let us now consider the isospin-polarized case. The neutron momentum distribution is affected by the scattering of both neutrons with neutrons $(n n)$ and neutrons with protons $(n p)$. Each of these is mediated by its own scattering scattering length. In the high-momentum limit, the distribution has the same structure as Eq. (A2) with $v=2$ :

$$
n_{n n}^{\gg}(k)=\frac{4 a_{n n}^{2} \rho_{n}}{k^{4}} .
$$

The scattering between different particles is somewhat different, as the internal integrals in Eq. (A1) need to take into account the different Fermi surfaces. With this in mind, the integral becomes

$$
n_{n p}(k)=\frac{v^{2}}{\left(2 \pi^{2}\right)^{3}} \frac{a_{n p}^{2}}{\rho_{n} m^{2}} \int_{k_{1}>k_{F}^{p}} d^{3} \mathbf{k}_{\mathbf{1}} \int_{k_{2}<k_{F}^{p}} d^{3} \mathbf{k}_{\mathbf{2}} \int_{k_{3}<k_{F}^{n}} d^{3} \mathbf{k}_{\mathbf{3}} \frac{\delta^{(3)}\left(\mathbf{k}+\mathbf{k}_{\mathbf{1}}-\mathbf{k}_{\mathbf{2}}-\mathbf{k}_{\mathbf{3}}\right)}{\left[\frac{k^{2}+k_{1}^{2}-k_{2}^{2}-k_{3}^{2}}{2 m}\right]^{2}} .
$$

Note that this contribution has a different overall degeneracy factor, associated with the lack of exchange in this channel. Taking again the $k \gg k_{F}^{\tau}$ limit, one finds

$$
n_{n p}^{\gg}(k)=\frac{8 a_{n p}^{2} \rho_{p}}{k^{4}} .
$$

Adding up the two contributions, we find the following expression for the high-momentum limit of the neutron momentum distribution:

$$
n_{n}^{\gg}(k ; \rho, \beta)=\frac{4}{k^{4}}\left[a_{n n}^{2} \rho_{n}+2 a_{n p}^{2} \rho_{p}\right] .
$$

Introducing the total density and the isospin-asymmetry parameter [Eq. (1)], one finds

$$
n_{n}^{\gg}(k ; \rho, \beta)=n^{\gg}(k ; \rho, 0) \times[1+\gamma \beta] .
$$

Here we have introduced the high-momentum limit in the symmetric case,

$$
n^{\gg}(k ; \rho, 0)=\frac{2 \rho}{k^{4}}\left[a_{n n}^{2}+2 a_{n p}^{2}\right] .
$$

An equivalent expression to Eq. (A7) with a minus sign holds for protons. The high-momentum components of the momentum distribution have the same $k^{-4}$ dependence as the unpolarized case [29]. Their overall prefactor, however, scales linearly with asymmetry. We have chosen to parametrize the isovector dependence of the scattering matrix in terms of a dimensionless parameter:

$$
\gamma=\frac{a_{n n}^{2}-2 a_{n p}^{2}}{a_{n n}^{2}+2 a_{n p}^{2}}
$$

For the ${ }^{1} S_{0}$ channel in $N N$ scattering, we find $\gamma=-0.52$.

Strictly speaking, however, the DFG model should not apply for nuclear systems around saturation densities. Nuclear scattering lengths are large compared to $k_{F}$, so that the expansion should break down in this regime. This signals the need to consider effective range effects as well as other many-body techniques. Moreover, the limit $k \gg 1$ is not necessarily of interest in our case, as the tensor-dominated region is not that far away from the Fermi surface itself around saturation.

Nevertheless, the DFG model is still a useful analytic tool that suggests scalings of correlations. If we take the results obtained here as a guiding principle for the scaling of momentum distributions with isospin asymmetry, we find that the functional form agrees well with our numerical results. We show the density dependence of the effective $\gamma$ parameter in dense matter in Fig. 5. Incidentally, the DFG $\gamma$ parameter has the same sign and order of magnitude than that in our dense matter calculations (see Fig. 5). In nuclear matter, we interpret this parameter as a measure of the strength of in-medium isovector correlation effects, rather than as a ratio of scattering lengths.
[1] C. Onderwater et al., Phys. Rev. Lett. 81, 2213 (1998).

[2] R. Starink et al., Phys. Lett. B 474, 33 (2000).

[3] D. Rohe et al., Phys. Rev. Lett. 93, 182501 (2004).

[4] E. Piasetzky, M. Sargsian, L. Frankfurt, M. Strikman, and J. W. Watson, Phys. Rev. Lett. 97, 162504 (2006).

[5] O. Benhar, I. Sick, and D. Day, Rev. Mod. Phys. 80, 189 (2008).

[6] R. Subedi et al., Science 320, 1476 (2008).

[7] J. Arrington, D. Higinbotham, G. Rosner, and M. Sargsian, Prog. Part. Nucl. Phys. 67, 898 (2012).

[8] W. U. Boeglin, L. Coman, P. Ambrozewicz, K. Aniol, J. Arrington, G. Batigne, P. Bosted, A. Camsonne, G. Chang, J. P. Chen, et al., Phys. Rev. Lett. 107, 262501 (2011).

[9] R. Amado, Phys. Rev. C 14, 1264 (1976).

[10] H. Müther, A. Polls, and W. H. Dickhoff, Phys. Rev. C 51, 3040 (1995).

[11] A. Rios, A. Polls, and W. H. Dickhoff, Phys. Rev. C 79, 064308 (2009).
[12] W. H. Dickhoff and D. V. Neck, Many-body Theory Exposed!, 2nd ed. (World Scientific, Singapore, 2008).

[13] W. H. W. Dickhoff and C. Barbieri, Prog. Part. Nucl. Phys. 52, 377 (2004).

[14] R. Schiavilla, R. B. Wiringa, S. C. Pieper, and J. Carlson, Phys. Rev. Lett. 98, 132501 (2007).

[15] L. B. Weinstein, E. Piasetzky, D. W. Higinbotham, J. Gomez, O. Hen, and R. Shneor, Phys. Rev. Lett. 106, 052301 (2011).

[16] J. Arrington, A. Daniel, D. B. Day, N. Fomin, D. Gaskell, and P. Solvignon, Phys. Rev. C 86, 065204 (2012).

[17] O. Hen, E. Piasetzky, and L. B. Weinstein, Phys. Rev. C 85, 047301 (2012).

[18] M. Vanhalst, J. Ryckebusch, and W. Cosyn, Phys. Rev. C 86, 044619 (2012).

[19] S. Fantoni and V. Pandharipande, Nucl. Phys. A 427, 473 (1984).

[20] A. Gezerlis and J. Carlson, Phys. Rev. C 81, 025803 (2010). 
[21] P. Grange, J. Cugnon, and A. Lejeune, Nucl. Phys. A 473, 365 (1987). M. Baldo, I. Bombaci, G. Giansiracusa, U. Lombardo, C. Mahaux, and R. Sartor, Phys. Rev. C 41, 1748 (1990). M. Baldo, G. Bomnaci I., Giansiracusa, U. Lombardo, C. Mahaux, and R. Sartor, Nucl. Phys. A 545, 741 (1992).

[22] P. Wang, S.-X. Gan, P. Yin, and W. Zuo, Phys. Rev. C 87, 014328 (2013).

[23] P. Yin, J.-Y. Li, P. Wang, and W. Zuo, Phys. Rev. C 87, 014314 (2013).

[24] L. Frankfurt, M. Sargsian, and M. Strikman, Int. J. Mod. Phys. A 23, 2991 (2008).

[25] M. McGauley and M. M. Sargsian, arXiv:1102.3973.

[26] M. M. Sargsian, Phys. Rev. C 89, 034305 (2014).

[27] M. Alvioli, C. Ciofi degli Atti, L. P. Kaptari, C. B. Mezzetti, and H. Morita, Phys. Rev. C 87, 034603 (2013).

[28] A. A. Abrikosov, L. P. Gorkov, and I. Y. Dzyaloshinskii, Quantum Field Theoretical Methods in Statistical Physics, 2nd ed. (Pergamon Press, Oxford, UK, 1965).

[29] R. Sartor and C. Mahaux, Phys. Rev. C 21, 1546 (1980); 25, 677 (1982).

[30] W. H. Dickhoff, C. C. Gearhart, E. P. Roth, A. Polls, and A. Ramos, Phys. Rev. C 60, 064319 (1999).

[31] R. B. Wiringa, V. G. J. Stoks, and R. Schiavilla, Phys. Rev. C 51, 38 (1995).

[32] R. Machleidt, F. Sammarruca, and Y. Song, Phys. Rev. C 53, R1483 (1996).

[33] D. R. Entem and R. Machleidt, Phys. Rev. C 68, 041001 (2003).

[34] A. Ekström, G. Baardsen, C. Forssén, G. Hagen, M. HjorthJensen, G. R. Jansen, R. Machleidt, W. Nazarewicz, T. Papenbrock, J. Sarich, et al., Phys. Rev. Lett. 110, 192502 (2013).

[35] R. B. Wiringa and S. C. Pieper, Phys. Rev. Lett. 89, 182501 (2002).

[36] M. Baldo, A. Polls, A. Rios, H.-J. Schulze, and I. Vidaña, Phys. Rev. C 86, 064001 (2012).
[37] A. Carbone, A. Cipollone, C. Barbieri, A. Rios, and A. Polls, Phys. Rev. C 88, 054326 (2013); A. Carbone, A. Polls, and A. Rios, ibid. 88, 044302 (2013).

[38] C. Drischler, V. Somà, and A. Schwenk, Phys. Rev. C 89, 025806 (2014)

[39] C. Xu and B.-A. Li, arXiv:1104.2075 [nucl-th].

[40] I. Vidaña, A. Polls, and C. Providência, Phys. Rev. C 84, 062801 (2011).

[41] A. Carbone, A. Polls, and A. Rios, Europhys. Lett. 97, 22001 (2012)

[42] W. Zuo, I. Bombaci, and U. Lombardo, Phys. Rev. C 60, 024605 (1999).

[43] T. Alm, B. Friman, G. Röpke, and H. Schulz, Nucl. Phys. A 551, 45 (1993).

[44] T. Frick, H. Müther, A. Rios, A. Polls, and A. Ramos, Phys. Rev. C 71, 014313 (2005).

[45] H. Müther and W. H. Dickhoff, Phys. Rev. C 72, 054313 (2005).

[46] T. Frick, Ph.D. thesis, University of Tübingen, 2004; A. Rios, Ph.D. thesis, University of Barcelona, 2007.

[47] B. E. Vonderfecht, W. H. Dickhoff, A. Polls, and A. Ramos, Phys. Rev. C 44, R1265 (1991).

[48] C. Mahaux and R. Sartor, Phys. Rep. 211, 53 (1992).

[49] N. Fomin, J. Arrington, R. Asaturyan, F. Benmokhtar, W. Boeglin, P. Bosted, A. Bruell, M. H. S. Bukhari, M. E. Christy, E. Chudakov, et al., Phys. Rev. Lett. 108, 092502 (2012).

[50] S. Bogner, R. Furnstahl, R. Perry, and A. Schwenk, Phys. Lett. B 649, 488 (2007).

[51] S. Bogner, R. Furnstahl, and A. Schwenk, Prog. Part. Nucl. Phys. 65, 94 (2010).

[52] S. K. Bogner and D. Roscher, Phys. Rev. C 86, 064304 (2012).

[53] I. Angeli and K. Marinova, At. Data Nucl. Data Tables 99, 69 (2013)

[54] H. Feldmeier, W. Horiuchi, T. Neff, and Y. Suzuki, Phys. Rev. C 84, 054003 (2011).

[55] K. Gubbels and H. Stoof, Phys. Rep. 525, 255 (2013).

[56] V. M. Galitskii, Sov. Phys.-JETP 7, 104 (1958). 